PROCEEDINGS OF THE

AMERICAN MATHEMATICAL SOCIETY

Volume 138, Number 2, February 2010, Pages 769-776

S 0002-9939(09)10072-2

Article electronically published on September 9, 2009

\title{
AMSTERDAM PROPERTIES OF WIJSMAN HYPERSPACES
}

\author{
JILING CAO AND HEIKKI J. K. JUNNILA
}

(Communicated by Alexander Dranishnikov)

\begin{abstract}
In this paper we show the following results: (i) there exists a separable metric space of the first category whose Wijsman hyperspace is almost countably subcompact; (ii) there exists a $\sigma$-discrete crowded metric space whose Wijsman hyperspace is countably base-compact. Neither of these can occur with Vietoris hyperspaces.
\end{abstract}

\section{INTRODUCTION}

In 1966, Wijsman [18 first considered the weak topology on the collection of nonempty closed subsets of a metric space, generated by the distance functionals viewed as functions of a set argument. Nowadays, this topology is known as the Wijsman topology. Since then, there has been a considerable effort in exploring various properties of this class of hyperspaces. For example, after Lechicki and Levi proved in 14 that the Wijsman hyperspace of a separable metric space is separable and metrizable, several topologists investigated the completeness of Wijsman hyperspaces of separable metric spaces. Already a much earlier result of Effros 9 can be interpreted as stating that a Polish space (i.e., a completely metrizable separable space) admits a metric for which the Wijsman topology is Polish. Later, Beer [3, 4] showed that the Wijsman hyperspace of any separable complete metric space is Polish; Costantini 7 completed this line of investigation by showing that, for a Polish space equipped with any compatible metric, the Wijsman hyperspace is Polish. Further, Costantini [8] constructed a (nonseparable) complete metric space whose Wijsman hyperspace is not Cech-complete (in this case, the Wijsman hyperspace is Tychonoff, but nonmetrizable). Since Čech-complete spaces are hereditarily Baire, investigating the Baire property of Wijsman hyperspaces comes into play. Recall that a topological space $X$ is Baire if the intersection of every sequence of dense open subsets in $X$ is dense, and if every nonempty closed subspace of $X$ is Baire, then $X$ is called hereditarily Baire. In this direction, Zsilinszky 20 showed that

Received by the editors February 3, 2009, and, in revised form, June 8, 2009.

2000 Mathematics Subject Classification. Primary 54E52; Secondary 54B10, 54B20.

Key words and phrases. Baire space, ball topology, pseudocomplete, subcompact, Wijsman topology.

Research for this paper was partially conducted during the first author's visit to the University of Helsinki in July 2008. He would like to acknowledge financial support from the Magnus Ehrnrooth Foundation, administered by the Finnish Society of Sciences and Letters, and he also thanks the Department of Mathematics and Statistics for hospitability.

The second author's research was partially supported by Natural Science Foundation of China grant 10671173. 
the Wijsman hyperspace of a complete metric space is Baire. Answering a question posed by Zsilinszky in [20], Chaber and Pol [6] showed that the Wijsman hyperspace of a complete metric space may fail to be hereditarily Baire, while Cao and Tomita [5] showed that the Wijsman hyperspace of a metric hereditarily Baire space is Baire. It is still an open problem whether the Wijsman hyperspace of every metric Baire space is Baire. All the aforementioned work concerns the question of whether the Wijsman hyperspace of a metric space $(X, d)$ possesses property $\mathcal{Q}$ when $(X, d)$ possesses property $\mathcal{P}$. Let us consider its inverse problem. Since a metric space $(X, d)$ is topologically embedded as a closed subspace into its Wijsman hyperspace, $(X, d)$ must be completely metrizable if its Wijsman hyperspace is Čech-complete. A result of $\mathrm{McCoy}$ in [16] asserts that for a $T_{1}$ topological space $X$, if its Vietoris hyperspace is Baire, then so is $X$. Thus, one would conjecture that if the Wijsman hyperspace of a metric space $(X, d)$ is Baire, then the space $(X, d)$ itself must be Baire. However, this is not the case. Recently, Pol and Zsilinszky showed that there is a metric space which is of the first category and whose Wijsman hyperspace is Baire 21]. This tells us that with respect to the Baire property, the Wijsman topology behaves differently from the Vietoris topology.

The main purpose of this paper is to continue the study of Wijsman hyperspaces in this direction. We show the following results: (i) there is a separable metric space of the first category whose Wijsman hyperspace is almost countably subcompact; and (ii) there is a $\sigma$-discrete crowded metric space whose Wijsman hyperspace is countably base-compact (recall that a topological space is crowded provided it has no isolated points). In the literature, almost countable subcompactness, countable base-compactness as well as a few other similar completeness properties are known as "Amsterdam properties". Since these properties are weaker than Čechcompleteness but stronger than the Baire property, our results sharpen those of Pol and Zsilinszky.

To proceed further, let us introduce some notation. For a given space $X$, let $2^{X}$ denote the family of nonempty closed subsets of $X$. For $E \subseteq X$, let $E^{-}=\{A \in$ $\left.2^{X}: A \cap E \neq \emptyset\right\}$ and $E^{+}=\left\{A \in 2^{X}: A \subseteq E\right\}$. The complement of $E$ (in $X$ ) is denoted by $E^{c}$ or $X \backslash E$. For a metric space $(X, d)$ and $x \in X$, the open (closed) ball centered at $x$ with radius $r$ is denoted by $S_{r}(x)\left(B_{r}(x)\right)$; that is,

$$
S_{r}(x)=\{y \in X: d(x, y)<r\} \text { and } B_{r}(x)=\{y \in X: d(x, y) \leq r\} .
$$

The Wijsman topology $\tau_{w(d)}$ on $2^{X}$ has

$$
\left\{U^{-}: U \in \tau(X)\right\} \cup\left\{\left\{A \in 2^{X}: d(x, A)>\varepsilon\right\}: x \in X, \varepsilon>0\right\}
$$

as a subbase. A topology closely related to $\tau_{w(d)}$ is the ball topology $\tau_{b(d)}$ on $2^{X}$, which has

$$
\left\{U^{-}: U \in \tau(X)\right\} \cup\left\{\left(B_{r}(x)^{c}\right)^{+}: x \in X, r>0\right\}
$$

as a subbase [4, 21. It is well known that $\tau_{w(d)} \subseteq \tau_{b(d)}$ holds on $2^{X}$ for any metric space $(X, d)$, 4, p. 53]. Regarding the Baire property, it was shown in 21] that for a metric space $(X, d),\left(2^{X}, \tau_{w(d)}\right)$ is Baire if and only if $\left(2^{X}, \tau_{b(d)}\right)$ is Baire. To state our examples, we need the Baire metric (also known as the first difference metric) defined on spaces of sequences. Given a cardinal $\kappa$, the Baire metric $d_{\kappa}$ on $\kappa^{\omega}$ is defined by

$$
d_{\kappa}(x, y)= \begin{cases}0, & \text { if } x=y \\ 2^{-n}, & \text { if } x \neq y \text { and } n \text { is the least with } x(n) \neq y(n) .\end{cases}
$$


Note that $\left(\kappa^{\omega}, d_{\kappa}\right)$ is an ultrametric space; that is, for any $x, y, z \in \kappa^{\omega}$,

$$
d_{\kappa}(x, z) \leq \max \left\{d_{\kappa}(x, y), d_{\kappa}(y, z)\right\} .
$$

Recall that ultrametric balls are clopen and satisfy the condition

(1.1) For any two balls $B_{1}$ and $B_{2}$ with $B_{1} \cap B_{2} \neq \emptyset$, either $B_{1} \subseteq B_{2}$ or $B_{2} \subseteq B_{1}$.

It is well known that $\left(\kappa^{\omega}, d_{\kappa}\right)$ is complete. Finally, we denote by $\left(\kappa^{\omega}\right)_{0}$ the set consisting of those elements of $\kappa^{\omega}$ which are eventually zero, endowed with the relative metric from $\left(\kappa^{\omega}, d_{\kappa}\right)$. For any undefined concepts and terminology, refer to the listed references.

\section{The Wijsman hyperspace of $\left(\omega^{\omega}\right)_{0} \times \omega^{\omega}$}

Let $X$ be a topological space. Recall that a collection $\mathcal{F}$ of nonempty subsets of $X$ is a regular filterbase if whenever $F_{1}, F_{2} \in \mathcal{F}$, there is an $F_{3} \in \mathcal{F}$ such that $\overline{F_{3}} \subseteq F_{1} \cap F_{2}$. The space $X$ is (almost) countably subcompact with respect to a $(\pi$-)base $\mathfrak{B}$ if $X$ is (quasi-)regular and $\bigcap\{\bar{B}: B \in \mathcal{B}\} \neq \emptyset$ for every countable regular filterbase $\mathcal{B} \subseteq \mathfrak{B},[2$. Evidently, every almost countably subcompact space is Baire.

Lemma 2.1 ([13]). A (quasi-)regular space $X$ is (almost) countably subcompact if and only if $X$ admits a ( $\pi$-)base $\mathfrak{B}$ such that $\bigcap_{n \in \mathbb{N}} B_{n} \neq \emptyset$ for every sequence $\left\{B_{n}: n \in \mathbb{N}\right\} \subseteq \mathfrak{B}$ satisfying $\overline{B_{n+1}} \subseteq B_{n}$ for all $n \in \mathbb{N}$.

Now, we consider the set $Y=\left(\omega^{\omega}\right)_{0} \times \omega^{\omega}$. We equip $Y$ with the metric $\rho$ defined by the formula

$$
\rho((r, t),(s, u))=\max \left\{d_{\omega}(r, s), d_{\omega}(t, u)\right\} .
$$

Then $\rho$ is an ultrametric, and the space $Y$ is separable. Moreover, $\left(\omega^{\omega}\right)_{0}$ is a countable space without isolated points, and it follows that $Y$ is of the first category. In 21, it was shown that the Wijsman hyperspace of $(Y, \rho)$ is Baire. In what follows, we shall sharpen this result by showing that the Wijsman hyperspace is almost countably subcompact. To this end, we first need a simple observation.

Lemma 2.2. If $(X, d)$ is a metric space such that for every point $x_{0} \in X$, the set $\left\{d\left(x_{0}, y\right): y \in X\right\}$ has no nonzero accumulation points in $\mathbb{R}^{+}$, then $\tau_{w(d)}$ and $\tau_{b(d)}$ coincide on $2^{X}$.

Proof. The conclusion follows directly from the following fact: under the hypothesis, for every $x_{0} \in X, A \in 2^{X}$, and $\varepsilon>0$, we have $A \cap B_{\varepsilon}\left(x_{0}\right)=\emptyset$ if and only if $d\left(x_{0}, A\right)>\varepsilon$.

Theorem 2.3. The hyperspace $\left(2^{Y}, \tau_{w(\rho)}\right)$ admits a $\pi$-base $\mathfrak{B}$ such that $\left(2^{Y}, \tau_{w(\rho)}\right)$ is almost countably subcompact with respect to $\mathfrak{B}$.

Proof. Note that, by Lemma 2.2 we have $\tau_{w(\rho)}=\tau_{b(\rho)}$ on $2^{Y}$. For each $n \in \mathbb{N}$, let $\mathcal{C}_{n}=\left\{B_{2^{-n}}(s): s \in \omega^{\omega}\right\}$. Note that each $\mathcal{C}_{n}$ is a partition of $\omega^{\omega}$ and that no member of $\mathcal{C}_{n}$ can be covered by finitely many members of the family $\bigcup_{k>n} \mathcal{C}_{k}$. Let $\mathcal{C}=\bigcup_{n \in \mathbb{N}} \mathcal{C}_{n}$. Note that $C \cap\left(\omega^{\omega}\right)_{0} \neq \emptyset$ for every $C \in \mathcal{C}$. Write $\left(\omega^{\omega}\right)_{0}=\{s(k)$ : $k \in \mathbb{N}\}$. For each $C \in \mathcal{C}$, we define $k_{C}=\min \{k \in \mathbb{N}: s(k) \in C\}$, and then we put $s_{C}=s\left(k_{C}\right)$. The following is clear.

(2.1) For all $C, D \in \mathcal{C}, s_{D}=s_{C}$ whenever $s_{C} \in D \subseteq C$. 
For each $n \in \mathbb{N}$, let $\mathcal{E}_{n}=\left\{\left(C \cap\left(\omega^{\omega}\right)_{0}\right) \times D: C, D \in \mathcal{C}_{n}\right\}$, and note that $\mathcal{E}_{n}$ is the family of all $\rho$-balls of radius $\frac{1}{2^{n}}$ in $Y$. Let $\mathcal{E}=\bigcup_{n \in \mathbb{N}} \mathcal{E}_{n}$, and for each $E \in \mathcal{E}$, define $n_{E}$ by the condition $E \in \mathcal{E}_{n_{E}}$. For each member $E=\left(C \cap\left(\omega^{\omega}\right)_{0}\right) \times D$ of the family $\mathcal{E}$, let $\widetilde{E}=\left\{s_{C}\right\} \times D$. For every $n \in \mathbb{N}$, denote by $\mathfrak{B}_{n}$ the family consisting of all nonempty sets of the form $\mathcal{U}=(Y \backslash \bigcup \mathcal{F})^{+} \cap \bigcap_{E \in \mathcal{A}} E^{-}$, where $\mathcal{F}$ is a finite subfamily of $\bigcup_{k=1}^{n} \mathcal{E}_{k}$ and $\mathcal{A}$ is a finite subfamily of $\mathcal{E}_{n}$ such that we have $\widetilde{G} \cap \bigcup \mathcal{A} \neq \emptyset$ whenever $G \in \mathcal{E}$ and $G \cap \bigcup \mathcal{A} \neq \emptyset$. Note that since $\mathcal{U}$ is nonempty and $\rho$ is an ultrametric, we have $\bigcup \mathcal{F} \cap \bigcup \mathcal{A}=\emptyset$. Let $\mathfrak{B}=\bigcup_{n \in \mathbb{N}} \mathfrak{B}_{n}$.

Claim. $\mathfrak{B}$ is a $\pi$-base for $\left(2^{Y}, \tau_{w(\rho)}\right)$.

Proof of the Claim. It is clear that every member of $\mathfrak{B}$ is in $\tau_{w(\rho)}$. Let $\mathcal{W}$ be an arbitrary nonempty $\tau_{w(\rho)}$-open set. Then there exist finite subfamilies $\mathcal{H}$ and $\mathcal{I}$ of $\mathcal{E}$ such that $(Y \backslash \cup \mathcal{H})^{+} \cap \bigcap_{E \in \mathcal{I}} E^{-}$is a nonempty basic open set contained in $\mathcal{W}$. Moreover, we can choose $\mathcal{H}$ and $\mathcal{I}$ so that $\bigcup \mathcal{H} \cap \bigcup \mathcal{I}=\emptyset$, and there exists $n \in \mathbb{N}$ such that $\mathcal{I} \subseteq \mathcal{E}_{n}$ and $\mathcal{H} \subseteq \bigcup_{k=1}^{n} \mathcal{E}_{k}$. To prove that $\mathcal{W}$ contains a member of $\mathfrak{B}$, we shall show that there exists a finite subfamily $\mathcal{A} \subseteq \mathcal{E}_{n}$ such that $\mathcal{I} \subseteq \mathcal{A}, \cup \mathcal{H} \cap \bigcup \mathcal{A}=\emptyset$ and $(Y \backslash \cup \mathcal{H})^{+} \cap \bigcap_{E \in \mathcal{A}} E^{-} \in \mathfrak{B}$. For this purpose, we let $\mathcal{E}_{n}^{\prime}=\left\{E \in \mathcal{E}_{n}: E \cap \bigcup \mathcal{H}=\emptyset\right\}$ and, further, $\mathcal{G}=\left\{G \in \mathcal{E}: E \subseteq G\right.$ for some $\left.E \in \mathcal{E}_{n}^{\prime}\right\}$. Note that we have $\mathcal{I} \subseteq \mathcal{E}_{n}^{\prime} \subseteq \mathcal{G}$. We show that for every $G \in \mathcal{G}$, there exists a set $J_{G} \in \mathcal{E}_{n}^{\prime}$ such that $J_{G} \subseteq G$ and $J_{G} \cap \widetilde{G} \neq \emptyset$. Let $E \in \mathcal{E}_{n}^{\prime}$ and $G \in \mathcal{E}$ be such that $E \subseteq G$. Then $n_{G} \leq n$. Since $E \cap \bigcup \mathcal{H}=\emptyset$, we have $G \nsubseteq \bigcup \mathcal{H}$. It follows from (1.1) that the family $\mathcal{H}_{G}=\{H \in \mathcal{H}: H \cap G \neq \emptyset\}$ is contained in the family $\bigcup_{m>n_{G}} \mathcal{E}_{m}$. Since no member of $\mathcal{C}_{n_{G}}$ is covered by finitely many members of $\bigcup_{m>n_{G}} \mathcal{C}_{m}$, the set $\widetilde{G}$ is not covered by $\mathcal{H}_{G}$ and hence not by $\mathcal{H}$ either. As a consequence, there exists $J_{G} \in \mathcal{E}_{n}$ such that $J_{G} \cap(\widetilde{G} \backslash \bigcup \mathcal{H}) \neq \emptyset$. Since $\mathcal{H} \subseteq \bigcup_{k=1}^{n} \mathcal{E}_{k}$ and $n_{G} \leq n$, by (1.1) again, we have $J_{G} \in \mathcal{E}_{n}^{\prime}$ and $J_{G} \subseteq G$. Note that we have $J_{G}=G$ for every $G \in \mathcal{E}_{n}^{\prime}$.

For each $E \in \mathcal{E}_{n}^{\prime}$, let $\mathcal{G}_{E}=\{G \in \mathcal{E}: E \subseteq G\}$ and $\mathcal{A}_{E}=\left\{J_{G}: G \in \mathcal{G}_{E}\right\}$; note that $\mathcal{G}_{E}$ is finite, $\mathcal{G}_{E} \subseteq \mathcal{G}$ and $E \in \mathcal{A}_{E} \subseteq \mathcal{E}_{n}^{\prime}$. Then the family $\mathcal{A}=\bigcup\left\{\mathcal{A}_{E}: E \in \mathcal{I}\right\}$ is finite and $\mathcal{I} \subseteq \mathcal{A} \subseteq \mathcal{E}_{n}^{\prime}$. Let $\mathcal{D}=(Y \backslash \cup \mathcal{H})^{+} \cap \bigcap_{E \in \mathcal{A}} E^{-}$. Since $\mathcal{I} \subseteq \mathcal{A}$, we have $\mathcal{D} \subseteq(Y \backslash \cup \mathcal{H})^{+} \cap \bigcap_{E \in \mathcal{I}} E^{-}$. It remains to show that $\mathcal{D} \in \mathfrak{B}$. First observe that, by construction, we have $\bigcup \mathcal{H} \cap \bigcup \mathcal{A}=\emptyset$. It follows that $Y \backslash \bigcup \mathcal{H} \in \mathcal{D}$ and hence $\mathcal{D} \neq \emptyset$. To complete the proof of $\mathcal{D} \in \mathfrak{B}$, let $G \in \mathcal{E}$ be such that $G \cap \bigcup \mathcal{A} \neq \emptyset$. We must show $\widetilde{G} \cap \bigcup \mathcal{A} \neq \emptyset$. Let $A \in \mathcal{A}$ be such that $A \cap G \neq \emptyset$. If $G \subseteq A$, then $\widetilde{G} \cap \bigcup \mathcal{A} \neq \emptyset$. Assume $G \nsubseteq \subseteq A$, and note that then $A \subseteq G$. In this case, we have $G \in \mathcal{G}_{A}$, and it follows that we have $J_{G} \in \mathcal{A}_{A} \subseteq \mathcal{A}$; again, we have $\widetilde{G} \cap \bigcup \mathcal{A} \neq \emptyset$. This completes the proof of $\mathcal{D} \in \mathfrak{B}$. We have shown that $\mathfrak{B}$ is a $\pi$-base for $\left(2^{Y}, \tau_{w(\rho)}\right)$.

To complete the proof of the theorem, let $\left\{\mathcal{B}_{k}: k \in \mathbb{N}\right\}$ be a sequence in $\mathfrak{B}$ such that $\mathcal{B}_{k+1} \subseteq \mathcal{B}_{k}$ for every $k \in \mathbb{N}$. We show $\bigcap_{k \in \mathbb{N}} \mathcal{B}_{k} \neq \emptyset$. For each $k \in \mathbb{N}$, let $n_{k}$ be such that $\mathcal{B}_{k} \in \mathfrak{B}_{n_{k}}$. We may assume (by passing to a subsequence, if necessary) that the sequence $\left\{n_{k}: k \in \mathbb{N}\right\}$ is nondecreasing. For each $k \in \mathbb{N}$, let $\mathcal{F}_{k} \subseteq \bigcup_{i=1}^{n_{k}} \mathcal{E}_{i}$ and $\mathcal{A}_{k} \subseteq \mathcal{E}_{n_{k}}$ be finite and such that $\mathcal{B}_{k}=\left(Y \backslash \cup \mathcal{F}_{k}\right)^{+} \cap \bigcap_{E \in \mathcal{A}_{k}} E^{-}$. Let $S=$ $Y \backslash \bigcup_{k \in \mathbb{N}} \cup \mathcal{F}_{k}$, and note that $S$ is a closed subset of $Y$. We show $S \in \bigcap_{k \in \mathbb{N}} \mathcal{B}_{k}$. Let $\ell \in \mathbb{N}$. According to the definition of the set $S$, we have $S \in\left(Y \backslash \cup \mathcal{F}_{\ell}\right)^{+}$. Hence the conclusion, that $S \in \mathcal{B}_{\ell}$, follows once we know that $S \cap E \neq \emptyset$ for every $E \in \mathcal{A}_{\ell}$. Let $E=\left(C \cap\left(\omega^{\omega}\right)_{0}\right) \times D \in \mathcal{A}_{\ell}$. We can inductively define a sequence $\left\{E_{k}: k \geq \ell\right\}$ with $E_{k} \in \mathcal{A}_{k}$ and $E_{k+1} \subseteq E_{k}$ for each $k \geq \ell$, as follows. First, we let $E_{\ell}=E$; if $E_{k} \in \mathcal{A}_{k}$ has already been defined for some $k \geq \ell$, then we can choose $E_{k+1} \in \mathcal{A}_{k+1}$ 
such that $E_{k+1} \cap \widetilde{E_{k}} \neq \emptyset$. This is possible, because it follows from the inclusion $\mathcal{B}_{k+1} \subseteq \mathcal{B}_{k}$ that the set $E_{k} \in \mathcal{A}_{k}$ must contain some member of $\mathcal{A}_{k+1}$ and then the definition of $\mathfrak{B}$ allows the existence of $E_{k+1} \in \mathcal{A}_{k+1}$ with $E_{k+1} \cap \widetilde{E_{k}} \neq \emptyset$. Write $E_{k}=\left(C_{k} \cap\left(\omega^{\omega}\right)_{0}\right) \times D_{k}$ for every $k \geq l$. For each $k$, we have $s_{C_{k}} \in C_{k+1} \subseteq C_{k}$, and it follows from (2.1) that $s_{C_{k+1}}=s_{C_{k}}$. As a consequence, there exists an $s \in\left(\omega^{\omega}\right)_{0}$ such that $s_{C_{k}}=s$ for every $k \geq \ell$. On the other hand, $\left\{D_{k}: k \geq \ell\right\}$ is a nested sequence of balls of the complete metric space $\left(\omega^{\omega}, d_{\omega}\right)$, and it follows from completeness and other properties of the ultrametric $d_{\omega}$ that there is a point $x \in \bigcap_{k \geq \ell} D_{k}$. Now we have $(s, x) \in \bigcap_{k \geq \ell} E_{k}$, and hence $(s, x) \in Y \backslash \bigcup_{k \geq \ell} \bigcup \mathcal{F}_{k}$. It is easy to see that we also have $(s, x) \in Y \backslash \bigcup_{k<\ell} \bigcup \mathcal{F}_{k}$. This is because $\mathcal{B}_{k+1} \subseteq \mathcal{B}_{k}$ implies $\bigcup \mathcal{F}_{k} \subseteq \bigcup \mathcal{F}_{k+1}$ for all $k \in \mathbb{N}$. As a consequence, we have $(s, x) \in S$. Since $(s, x) \in E_{\ell}=E$, we have shown $S \cap E \neq \emptyset$.

Recall that a topological space $X$ is said to be pseudocomplete [17] if $X$ is quasiregular and has a sequence $\left\{\mathfrak{B}_{n}: n \in \mathbb{N}\right\}$ of $\pi$-bases such that $\bigcap_{n \in \mathbb{N}} V_{n} \neq \emptyset$ whenever $\overline{V_{n+1}} \subseteq V_{n} \in \mathfrak{B}_{n}$ for each $n \in \mathbb{N}$. Clearly, every almost countably subcompact space is pseudocomplete.

Corollary 2.4. The Wijsman hyperspace of $(Y, \rho)$ is pseudocomplete.

Corollary 2.5. The Wijsman hyperspace of $(Y, \rho)$ has a dense completely metrizable subspace.

Proof. According to [14, Theorem 3.5], the Wijsman hyperspace of the metric space $(Y, \rho)$ is metrizable. On the other hand, it is shown in [1, Corollary 2.4] that a metrizable space is pseudocomplete if and only if the space has a dense completely metrizable subspace. Thus, the conclusion follows from Corollary 2.4.

Theorem 2.3 suggests the question of whether the Wijsman hyperspace of the space $(Y, \rho)$ is countably subcompact. However, this question has a negative answer, since it turns out that no non-Polish separable metrizable space admits a countably subcompact Wijsman hyperspace. To see this, let $(X, d)$ be a separable metric space with a countably subcompact Wijsman hyperspace. The Wijsman hyperspace of the separable space $(X, d)$ is separable and metrizable [14], and de Groot [12] has shown that countably subcompact metrizable spaces are completely metrizable. It follows that the Wijsman hyperspace of $(X, d)$ is Polish. This implies that $X$ is Polish, since $\left(2^{X}, \tau_{w(d)}\right)$ contains a closed copy of $X$. Nevertheless, if we consider nonseparable spaces, then even a metric space of the first category can have a countably subcompact Wijsman hyperspace, as we shall see in the next section.

\section{The Wijsman hyperspace of $\left(\kappa^{\omega}\right)_{0}$ FOR $\kappa \geq \omega_{1}$}

Recall that a space $X$ is (almost) countably base-compact with respect to an open $\left(\pi\right.$-) base $\mathfrak{B}$ if $X$ is (quasi-)regular such that $\bigcap_{F \in \mathcal{F}} \bar{F} \neq \emptyset$ for each countable centered family $\mathcal{F} \subseteq \mathfrak{B}$; see [2]. By definition, every (almost) countably base-compact space is (almost) countably subcompact.

In this section, we show that the Wijsman hyperspace of $\left(\left(\kappa^{\omega}\right)_{0}, d_{\kappa}\right)$ is countably base-compact when $\kappa \geq \omega_{1}$.

Theorem 3.1. Let $(X, d)$ be an ultrametric space such that no d-ball is covered by countably many smaller d-balls. Then $\left(2^{X}, \tau_{b(d)}\right)$ is countably base-compact. 
Proof. Denote by $\mathcal{E}$ the family consisting of all open $d$-balls, and denote by $\mathfrak{B}$ the (standard) base of $\tau_{b(d)}$ consisting of all sets $\mathcal{B} \subseteq 2^{X}$ of the form $\mathcal{B}=(X \backslash \cup \mathcal{F})^{+} \cap$ $\bigcap_{E \in \mathcal{A}} E^{-}$, where $\mathcal{F}$ and $\mathcal{A}$ are finite subfamilies of $\mathcal{E}$. We show that $\left(2^{X}, \tau_{b(d)}\right)$ is countably base-compact with respect to $\mathfrak{B}$. Let $\left\{\mathcal{B}_{k}: k \in \mathbb{N}\right\}$ be a countable centered subfamily of $\mathfrak{B}$. For every $k \in \mathbb{N}$, let $\mathcal{F}_{k}$ and $\mathcal{A}_{k}$ be finite subfamilies of $\mathcal{E}$ such that $\mathcal{B}_{k}=\left(X \backslash \bigcup \mathcal{F}_{k}\right)^{+} \cap \bigcap_{E \in \mathcal{A}_{k}} E^{-}$. Let $S=X \backslash \bigcup_{k \in \mathbb{N}} \bigcup \mathcal{F}_{k}$, and note that $S$ is a closed subset of $X$. We show that $S \in \bigcap_{k \in \mathbb{N}} \mathcal{B}_{k}$. Let $\ell \in \mathbb{N}$. According to the definition of the set $S$, we have $S \in\left(X \backslash \bigcup \mathcal{F}_{\ell}\right)^{+}$; hence, the conclusion that $S \in \mathcal{B}_{\ell}$ follows once we show that $S \cap E \neq \emptyset$ for every $E \in \mathcal{A}_{\ell}$. Let $E \in \mathcal{A}_{\ell}$. For every $k \in \mathbb{N}$, since $\mathcal{B}_{k} \cap \mathcal{B}_{\ell} \neq \emptyset$, we have $E \nsubseteq \subseteq \cup \mathcal{F}_{k}$, and it follows that, for every $F \in \mathcal{F}_{k}$, either $F$ is disjoint from $E$ or $F$ is a proper subset of $E$. Since the $d$-ball $E$ is not covered by countably many smaller $d$-balls, we have $E \nsubseteq \bigcup_{k \in \mathbb{N}} \cup \mathcal{F}_{k}$. Hence, the set $E \cap S=E \backslash \bigcup_{k \in \mathbb{N}} \bigcup \mathcal{F}_{k}$ is nonempty. This completes the proof that $S \in \bigcap_{k \in \mathbb{N}} \mathcal{B}_{k}$. We have shown that $\left(2^{X}, \tau_{b(d)}\right)$ is countably base-compact with respect to $\mathfrak{B}$.

Theorem 3.1 and Lemma 2.2 have the following consequence.

Corollary 3.2. Let $(X, d)$ be an ultrametric space such that no $d$-ball is covered by countably many smaller $d$-balls and no set $\{d(y, x): y \in X\}$, where $x \in X$, has nonzero accumulation points in $\mathbb{R}^{+}$. Then $\left(2^{X}, \tau_{w(d)}\right)$ is countably base-compact.

Remark 3.3. (i) Note that we have above countable base-compactness in a strong form: we have $\bigcap \mathfrak{C} \neq \emptyset$ for every countable linked subfamily $\mathfrak{C} \subseteq \mathfrak{B}$ (the family $\mathfrak{C}$ is linked if any two members of $\mathfrak{C}$ intersect).

(ii) Since the base $\mathfrak{B}$ in the proof above consists of clopen sets, we can actually strengthen countably base-compact to countably regularly co-compact.

Example 3.4. For any cardinal $\kappa \geq \omega_{1}$, consider the metric space $\left(\left(\kappa^{\omega}\right)_{0}, d_{\kappa}\right)$. It can be easily checked that $\left(\left(\kappa^{\omega}\right)_{0}, d_{\kappa}\right)$ satisfies the assumptions of Corollary 3.2. As a consequence, the hyperspace $\left(2^{\left(\kappa^{\omega}\right)_{0}}, \tau_{w\left(d_{\kappa}\right)}\right)$ is countably base-compact. On the other hand, for each $n<\omega$, if we set

$$
F_{n}=\left\{x \in\left(\kappa^{\omega}\right)_{0}: x(i)=0 \text { when } i \geq n\right\},
$$

then $F_{n}$ is a discrete subspace of $\left(\left(\kappa^{\omega}\right)_{0}, d_{\kappa}\right)$. Since $\left(\kappa^{\omega}\right)_{0}=\bigcup_{n<\omega} F_{n}$, the space $\left(\left(\kappa^{\omega}\right)_{0}, d_{\kappa}\right)$ is $\sigma$-discrete. It follows, since $\left(\left(\kappa^{\omega}\right)_{0}, d_{\kappa}\right)$ is a crowded space, that $\left(\left(\kappa^{\omega}\right)_{0}, d_{\kappa}\right)$ is of the first category.

\section{Further REMARKS AND OPEN QUESTIONS}

The Pol-Zsilinszky example and Example 3.4 show that the Wijsman hyperspace of a metric space can be of the second category even if the space is either separable and of the first category or $\sigma$-discrete and crowded. Our last result shows that these two examples cannot be simultaneously generalized; i.e., there is no countable crowded metric space with a second category Wijsman hyperspace. First we observe that the Wijsman topology is of the first category if and only if the same holds for the ball topology. Recall that a mapping $f: X \rightarrow Y$ is said to be feebly continuous [1] if $\operatorname{int}\left(f^{-1}(V)\right) \neq \emptyset$ whenever $f^{-1}(V) \neq \emptyset$. Let $(X, d)$ be a metric space. As noted in [19, proof of Theorem 5.1], the identity mapping Id : $\left(2^{X}, \tau_{w(d)}\right) \rightarrow\left(2^{X}, \tau_{b(d)}\right)$ is open and feebly continuous. Since feeble homeomorphisms map nowhere dense sets onto nowhere dense sets, $\left(2^{X}, \tau_{w(d)}\right)$ is of the first category if and only if $\left(2^{X}, \tau_{b(d)}\right)$ is of the first category. 
Proposition 4.1. Let $(X, d)$ be a $\sigma$-compact metric space of the first category. Then $\left(2^{X}, \tau_{w(d)}\right)$ is of the first category.

Proof. Let $X=\bigcup_{n \in \mathbb{N}} K_{n}$, where each $K_{n}$ is compact and nowhere dense in $(X, d)$. Then $2^{X}=\bigcup_{n \in \mathbb{N}} K_{n}^{-}$. Let $n \in \mathbb{N}$. We show that $K_{n}^{-}$is nowhere dense in $\left(2^{X}, \tau_{b(d)}\right)$. Assume not. Then there exists a nonempty finite family $\mathcal{G}$ of nonempty open subsets of $X$ and a family $\mathcal{F}$ of closed $d$-balls such that $\bigcup \mathcal{F} \cap \bigcup \mathcal{G}=\emptyset$ and the set $\mathcal{B}=(X \backslash \bigcup \mathcal{F})^{+} \cap \bigcap_{G \in \mathcal{G}} E^{-}$is contained in $K_{n}^{-}$. For each $G \in \mathcal{G}$, since $K_{n}$ is nowhere dense, there exists a point $p_{G} \in G \backslash K_{n}$. Now the set $S=\left\{p_{G}: G \in \mathcal{G}\right\}$ belongs to $\mathcal{B}$ but not to $K_{n}^{-}$, a contradiction. By the foregoing, we conclude that $\left(2^{X}, \tau_{b(d)}\right)$, and thus $\left(2^{X}, \tau_{w(d)}\right)$, is of the first category.

The following questions ask about possible improvements in the results of this paper.

Question 4.2. Can the conclusion of Theorem 2.3 be strengthened to almost countable base-compactness?

Question 4.3. Can the conclusion of Theorem 3.1 be strengthened to base compactness? In particular, is $\left(2^{\left(\omega_{1}^{\omega}\right)_{0}}, \tau_{w\left(d_{\omega_{1}}\right)}\right)$ base-compact?

Question 4.4. Does $\left(2^{\left(\omega_{1}^{\omega}\right)_{0}}, \tau_{w\left(d_{\omega_{1}}\right)}\right)$ have a dense completely metrizable subspace?

In the Introduction we mentioned the problem of whether the Wijsman hyperspace of every metric Baire space is Baire. Our results may have some bearing on this problem. If one tries to think about a possible Baire metric space with a non-Baire-Wijsman hyperspace, the first spaces to be considered would perhaps be the barely Baire spaces of Fleissner and Kunen in [10. However, all those spaces from [10] are ultrametric uniformly nowhere locally separable spaces of the kind considered in Corollary 3.2, so the corollary rules out the possibility of using these barely Baire spaces as counterexamples to the problem whether Baireness of $(X, d)$ implies that of $\left(2^{X}, \tau_{w(d)}\right)$. One can also ask whether some properties stronger than Baireness are inherited by Wijsman hyperspaces.

Question 4.5. If $(X, d)$ is a pseudocomplete (subcompact, base-compact) metric space, must its Wijsman hyperspace be pseudocomplete (subcompact, basecompact)?

Since the Wijsman hyperspace of a metric space $(X, d)$ can be embedded into the function space $C_{p}(X)$, a recent result of Lutzer, van Mill, and Tkachuk might be of some interest in this connection. They proved in [15] that $C_{p}(X)$ is subcompact only when $X$ is discrete.

\section{ACKNOWLEDGEMENT}

We thank Aarno Hohti for stimulating discussions on the topic of hyperspaces.

\section{REFERENCES}

1. J. M. Aarts and D. J. Lutzer, Pseudo-completeness and the product of Baire spaces, Pacific J. Math. 48 (1973), 1-10. MR0326666 (48:5009)

2. J. M. Aarts and D. J. Lutzer, Completeness properties designed for recognizing Baire spaces, Dissertationes Math. 116 (1974), 1-48. MR0380745 (52:1642)

3. G. Beer, A Polish topology for the closed subsets of a Polish space, Proc. Amer. Math. Soc. 113 (1991), 1123-1133. MR1065940 (92c:54009) 
4. G. Beer, Topologies on closed and closed convex sets, Kluwer, Dordrecht, 1993. MR:1269778 (95k:49001)

5. J. Cao and A. H. Tomita, The Wijsman hyperspace of a metric hereditarily Baire space is Baire, Topology Appl., to appear.

6. J. Chaber and R. Pol, Note on the Wijsman hyperspaces of completely metrizable spaces, Boll. Unione Mat. Ital. Sez. B Artic. Ric. Mat. (8) 5 (2002), 827-832. MR1934383 (2004j:54013)

7. C. Costantini, Every Wijsman topology relative to a Polish space is Polish, Proc. Amer. Math. Soc. 123 (1995), 2569-2574. MR.1273484 (95j:54012)

8. C. Costantini, On the hyperspace of a non-separable metric space, Proc. Amer. Math. Soc. 126 (1998), 3393-3396. MR 1618729 (99e:54006)

9. E. G. Effros, Convergence of closed subsets in a topological space, Proc. Amer. Math. Soc. 16 (1965), 929-931. MR0181983 (31:6208)

10. W. G. Fleissner and K. Kunen, Barely Baire spaces, Fund. Math. 101 (1978), 229-240. MR:521125 (80f:54009)

11. Z. Frolík, Remarks concerning the invariance of Baire spaces under mappings, Czech. Math. J. 11 (1960), 381-385. MR.0133098 (24:A2932)

12. J. de Groot, Subcompactness and the Baire category theorem, Indag. Math. 25 (1963), 761767. MR0159303 (28:2520)

13. Y. Ikeda, Cech-completeness and countable subcompactness, Topology Proc. 14 (1989), 75-87. MR 1081121 (92b:54044)

14. A. Lechicki and S. Levi, Wijsman convergence in the hyperspace of a metric space, Boll. Un. Mat. Ital. B (7) 1 (1987), 439-451. MR896334 (88e:54007)

15. D. J. Lutzer, J. van Mill and V. V. Tkachuk, Amsterdam properties of $C_{p}(X)$ imply discreteness of X, Canad. Math. Bull. 51 (2008), 570-578. MR2462461 (2009g:54011)

16. R. A. McCoy, Baire spaces and hyperspaces, Pacific J. Math. 58 (1975), 133-142. MR0410689 $(53: 14436)$

17. J. C. Oxtoby, Cartesian products of Baire spaces, Fund. Math. 49 (1961), 157-166. MR $0140638(25: 4055)$

18. R. Wijsman, Convergence of sequences of convex sets, cones and functions II, Trans. Amer. Math. Soc. 123 (1966), 32-45. MR0196599 (33:4786)

19. L. Zsilinszky, Baire spaces and hyperspace topologies, Proc. Amer. Math. Soc. 124 (1996), 2575-2584. MR1343733 (96j:54017)

20. L. Zsilinszky, Polishness of the Wijsman topology revisited, Proc. Amer. Math. Soc. 126 (1998), 3763-3735. MR.1458275 (99b:54014)

21. L. Zsilinszky, On Baireness of the Wijsman hyperspace, Boll. Unione Mat. Ital. Sez. B Artic. Ric. Mat. (8) 10 (2007), 1071-1079.

School of Computing and Mathematical Sciences, Auckland University of Technology, Private Bag 92006, Auckland 1142, New Zealand

E-mail address: jiling.cao@aut.ac.nz

Department of Mathematics and Statistics, The University of Helsinki, P. O. Box 68, FI-00014, Helsinki, Finland

E-mail address: heikki.junnila@helsinki.fi 